\title{
A Study of Boundary Areas in Office Space
}

\author{
Masahiro Saji*1 ${ }^{1}$, Mami Kurita ${ }^{2}$, Yuji Matsumoto ${ }^{3}$, Ryusuke Naka ${ }^{4}$ and Shigeyuki Yamaguchi ${ }^{5}$ \\ Graduate Student, Graduate school of Design Engineering and Management, Kyoto Institute of Technology, Japan \\ ${ }^{2}$ Kawakami Architectural Design Room, Japan \\ ${ }^{3}$ Research Associate, Department of Design Engineering and Management, Kyoto Institute of Technology, Japan \\ ${ }^{4}$ Associate Professor, Department of Design Engineering and Management, Kyoto Institute of Technology, Japan \\ ${ }^{5}$ Professor, Department of Design Engineering and Management, Kyoto Institute of Technology, Japan
}

\begin{abstract}
The aim of this study is to find the architectural composition for "boundary areas" in office space. As a first step to reach this aim, this paper focuses on the relationship between architectural planning factors for boundary areas and workers' activities in the office. In this paper we have defined "boundary area" as an area that has no particular function for office work and exists between individual functional spaces. The methods used in this study are listed below.

1. We have examined recent advanced offices in order to investigate a trend for the boundary areas.

2. A case study was conducted in an office focusing on activities that occurred in the boundary areas.

In the first study, we found that the zone level boundary area is one of the trend factors for planning recent offices. And we also discovered that the architectural characteristic of the zone level boundary areas could be classified into three categories: "absorber type", "buffer type" and "passage type". In the second study, we confirmed that valuable communication exchanges for office work were activated in buffer type boundary areas. From this case study and former studies, the relations between office planning factors for the boundary areas and communication activities which took place in these areas have been verified through the table in our conclusion.
\end{abstract}

Keywords: office planning; communications; activity; work place; boundary areas

\section{Introduction}

ICT (Information and Communication Technology) enables people to work interactively at various places including outside of office buildings. For example, a new work-style called mobile-work or nomad-work came into being due to the downsizing of personal computers. In fact, communication and collaboration between distributed workers has become possible. From the organizational point of view, it is important to relate with workers from other sections and groups or professionals outside the company. Consequently, work-style for employees has been changing and some new types of physical workspace have been proposed. Non-territorial offices and Satellite offices are some notable examples.

One of the important issues for office planning is 'how the workplace creates communication between workers'. Especially with regards to collaborative work, communication is the essential factor for running projects, creating new ideas and solving problems. The

\footnotetext{
*Contact Author: Masahiro Saji, Graduate Student,

Graduate school of Design Engineering and Management, Kyoto Institute of Technology, 1 Matsugasakihashikamicho Sakyou-ku, Kyoto-shi 606-8585 Japan

Tel: +81-75-705-2338 Fax: +81-75-705-2338

e-mail: masahiro_saji@nifty.com

(Received October 11, 2005 ; accepted February 2, 2006)
}

existing office planning methods based on "blocking" and "stacking" are not enough to create new office space, because these methods are applied to "optimize area" by dividing or grouping individual functional space for work processes. Our approach in this study is to design the relationship between each functional space in the office. This paper discusses the importance of the boundary areas in the office space and makes clear the workers' activities that occurred in these areas.

\section{Composition of Office Space and the Definition of Boundary Areas}

The volume of office space can be classified into six levels: 1. Building level, 2. Floor level, 3. Function level, 4. Room level, 5. Zone level and 6. Work station level. (Fig.1.) Each office space is distributed by floors and walls, and integrated by elevators and corridors. In this study, we define a boundary area as an area that has no particular function for office work and exists between individual functional spaces. For example, a corridor that is generally designed for connecting rooms is not a boundary area. But if the corridor becomes wide enough to set tables and bookshelves, it would be possible to call this space a meeting place. It can be used liberally as a boundary area. This is just one example of how to utilize space for a boundary area. The boundary area can be available for various functions. 


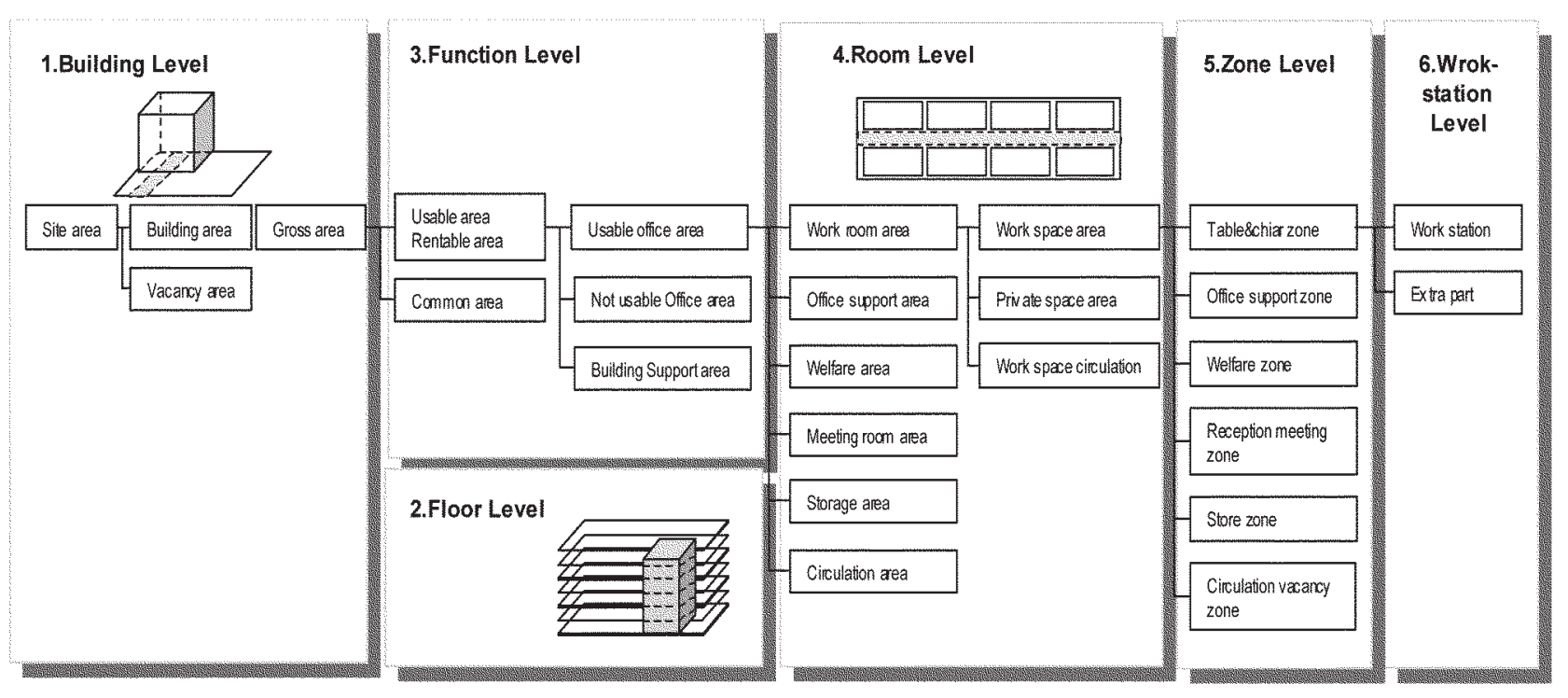

Fig.1. Composition of the Office Space

\section{Layout Trends for Boundary Areas}

In order to investigate layout trends for Boundary areas, we take 182 cases of advanced offices in this study. These 182 cases were collected between 1999 to 2004 from the "Nikkei New Office Award" which was organized by the "New Office Promotion Association" in Japan.

We examined the office planning and reported the concepts to the "Nikkei New Office Award". The number of cases applied in boundary areas was counted for each level in order to examine the tendency of office layouts for the boundary areas. The "layout rate" in Fig.2. is calculated so that the number of cases applied in the boundary area for each level is divided by the total number of cases in each year.

As a result, the layout rate of the building level has the highest ratio, which can explain the fact that the entrance to the ground floor of a building, for example, is generally a place where one does not have a clear function for office work. Especially if the building is taller and has a massive capacity, the entrance needs to be a foyer for receptions, meeting space and waiting area outside elevators instead of an office space. Therefore, the building level is utilized positively as a boundary area between the space that is the inside and outside of the building.

What we noticed is that the layout rate of zone levels in 2004 was about five times higher than 2000 and increased year after year. This may indicate the importance of the zone level boundary areas. There are many open space offices that exist in Japan. In addition, the aim of recent office planning and designing has been changing from expressing façade form or characteristic of a company's organization to supporting activities of workers and teams.

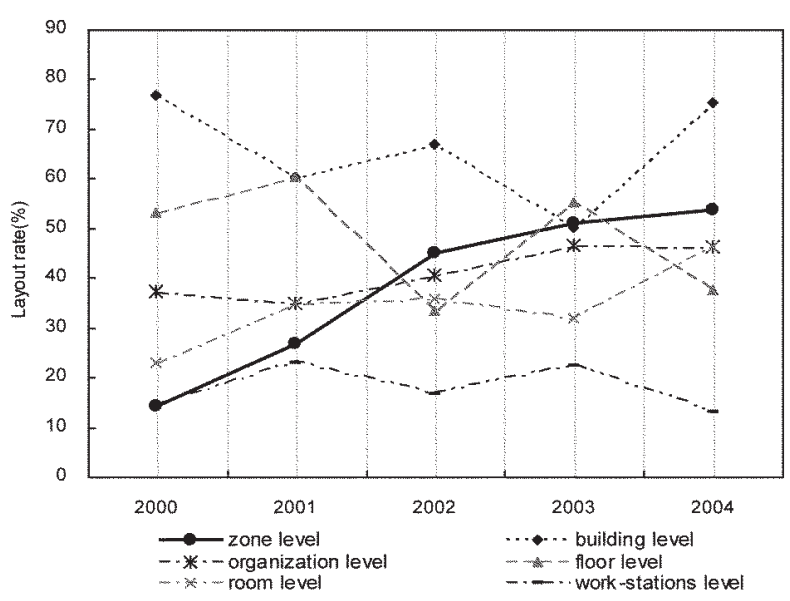

Fig.2. Boundary Area Trends in Japan

\section{Categories for the Zone Level Boundary Areas}

Because of the importance of the zone level boundary areas in the previous study, the 69 cases involving the zone level boundary areas were carefully examined. We collected data on purposes of office planning concepts for the cases reported to the competition, and then characterized them into three categories of "absorber type", "buffer type" and "passage type". (Table 1.)

In order to find the architectural planning factors for the zone level boundary areas, we classified them into three steps: a) Layout patterns and office furniture, b) Articulation of space, and c) Integration of space.

Following these steps, we made clear the details of the architectural characteristics in each type of the zone level boundary areas. This was done by comparing the ratio of the total number of offices utilized by the architectural factor and then dividing them by the number of cases in each type. 
Table 1. Zone Level Boundary Area Planning Concepts

\begin{tabular}{l|l|l}
\hline \multicolumn{1}{c|}{ Types } & \multicolumn{1}{c|}{ Characteristics of planning concept } & The number of case \\
\hline Absorber & Absorbing people, and supporting their communication in meetings & 22 \\
\hline Buffer & Flexible work setting suited for the circumstances of work process and team condition. & 21 \\
\hline Passage & Supporting ad hoc communication in middle of traffic line & 26 \\
\hline
\end{tabular}

a) Layout Patterns and Office Furniture

\section{- Layout Patterns}

The layout types that have been classified into five patterns depending on the relation when adjoining zones are compared with the ratio. (Fig.3.) According to this result, the passage type shows a high ratio on the layout pattern No. 2. The buffer type reveals a high ratio on the layout pattern No. 4. Additionally, the buffer type takes more than $70 \%$ of the sum of pattern No. 4 and No. 5, which is the layout plan surrounded by the other zone. This is extended to cover the notion of a multiplicity of activities taken in the area from other neighbor zones as one of the planning factors.

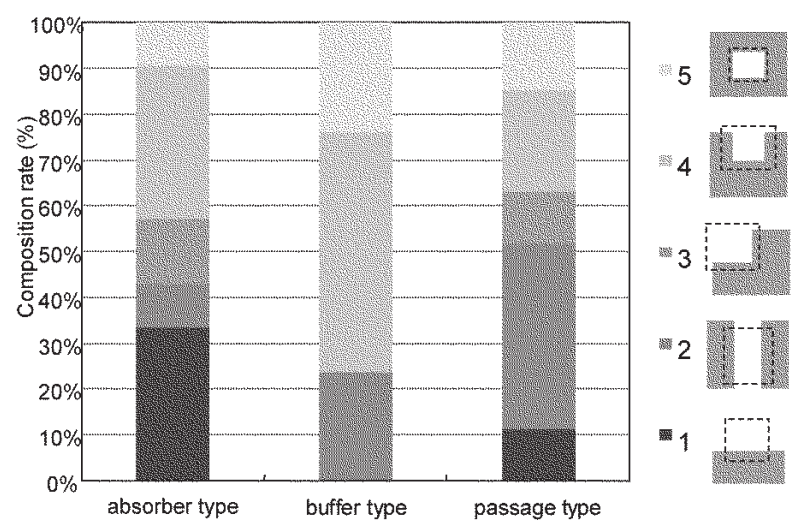

Fig.3. Layout Patterns

\section{- Office Furniture}

The office furniture organization is divided into two patterns: the concentration pattern that serves at one place with the same office furniture and the spread pattern that serves at various places with a variety of office furniture. (Fig.4.) The spread pattern shows higher ratio in all boundary area types, especially in the buffer type. The flexible office furniture planning such as spread pattern can be important as the planning factor for the buffer type boundary areas.

\section{b) Articulation of Space}

\section{- Articulation set Vertically}

As a way to articulate vertically as shown in Fig.5., the architectural factors are determined by three concerns: 1. Stick factors like pillars, 2. Surface factors like walls, and 3. Volume issues like filing cabinets.

In comparison with the ratio of these factors, the absorber type has high ratio for surface factors, in other words, it articulates a space clearly. On the other hand, the high ratio of volume that obscures the articulation of a space is in the passage type.

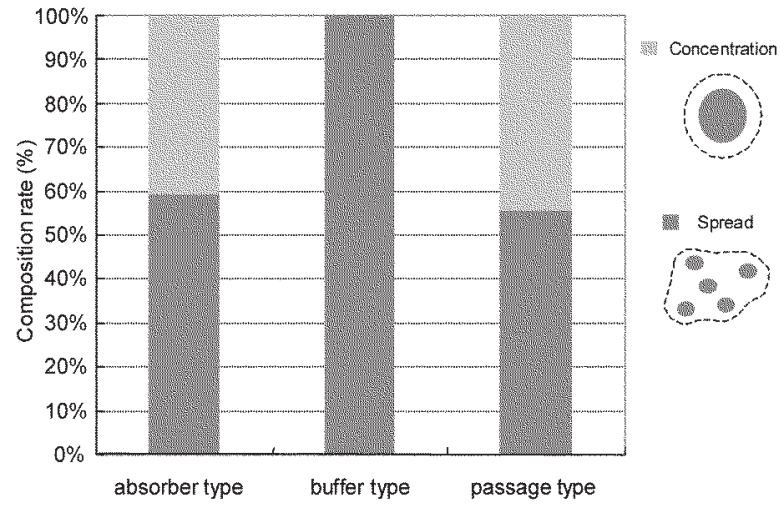

Fig.4. Office Furniture Organization

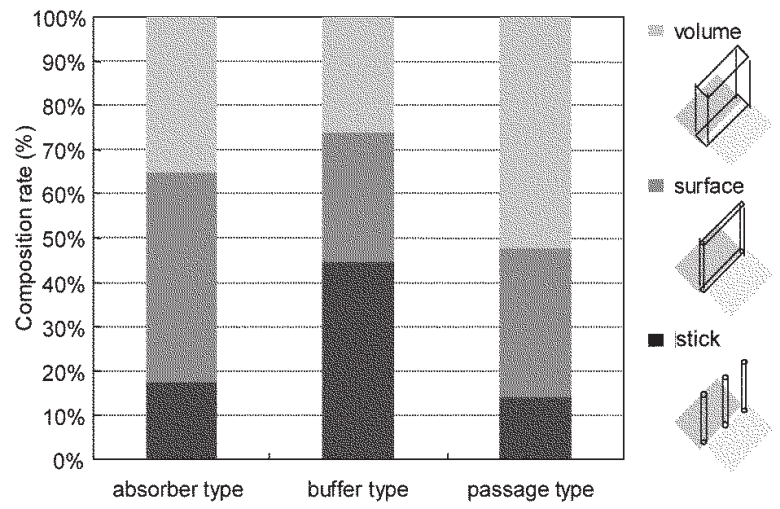

Fig.5. Articulation Set Vertically

\section{- Articulation set Horizontally}

As a way to articulate horizontally as shown in Fig.6., the architectural factors can also be determined by three factors: 1. Roof level, 2. Floor level, and 3. Materials on the floor.

According to this result, the ratio for the buffer type is similar to the passage type with a high ratio for the roof level and materials on the floor factors.

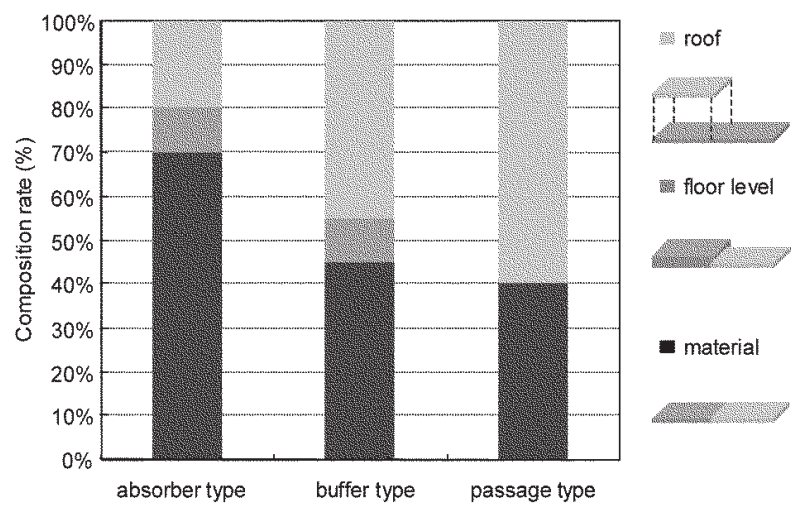

Fig.6. Articulation Set Horizontally 
Considering the ratio of "stick factors" for buffer type in vertical analysis, the buffer type may give a sense of being surrounded in this area.

\section{c) The Integration of Space}

\section{- Integration by Traffic Lines}

The traffic lines are divided into levels in terms of primary traffic lines, and secondly traffic lines and third traffic lines. (Fig.7.) We analyzed which level of traffic lines were involved in each boundary area.

The ratio of the third traffic line in the absorber type boundary area is much higher than the others so that this area is located at the deep circulation level for the planning factor.

The point in the line chart indicates where more than two parts of bar chart meet. Over $60 \%$ of the ratio where traffic lines meet is accounted for in the buffer type boundary areas, so that the planning factor for this area can be characterized as the combined traffic line of the circulation level.

\section{- Integration by Sight}

The space is judged by the combination of border types: "continuity", "indication", and "barrier". (Fig.8.) And then the space is classified into three categories as listed below.

- Continuous space:

A space which is composed of only continuous types.

- Adjustable continuous space:

A place which is composed of only indication types or a combination of indication types and continuous types. And a space that is composed of all three types.

- Restricted continuous space:

A space which is composed of only barrier types or a combination of barrier types and one of the other types.

As Fig.8. indicates, the ratio of continuous space is particularly high in the passage type. And the buffer type accounts for $60 \%$ of the ratio in adjustable continuous space. Therefore, the passage type boundary area has good visibility, and the buffer type has an adjustable continuous visibility as the planning factor.

\section{Planning Factors for Zone Level Boundary Area}

As a result of previous studies, the boundary areas have been classified and characterized according to the planning factors. (Table 2.)

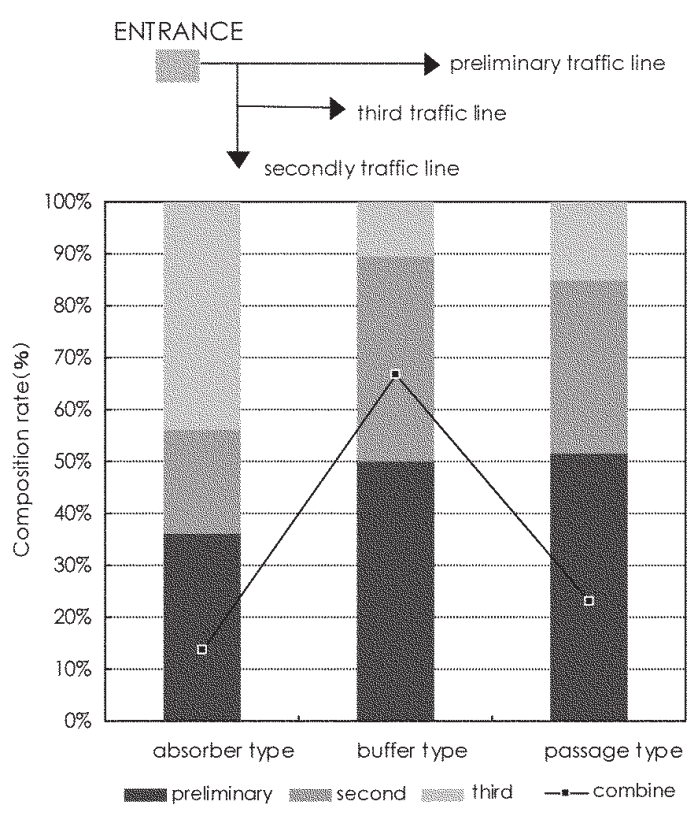

Fig.7. Integration by the Traffic Line
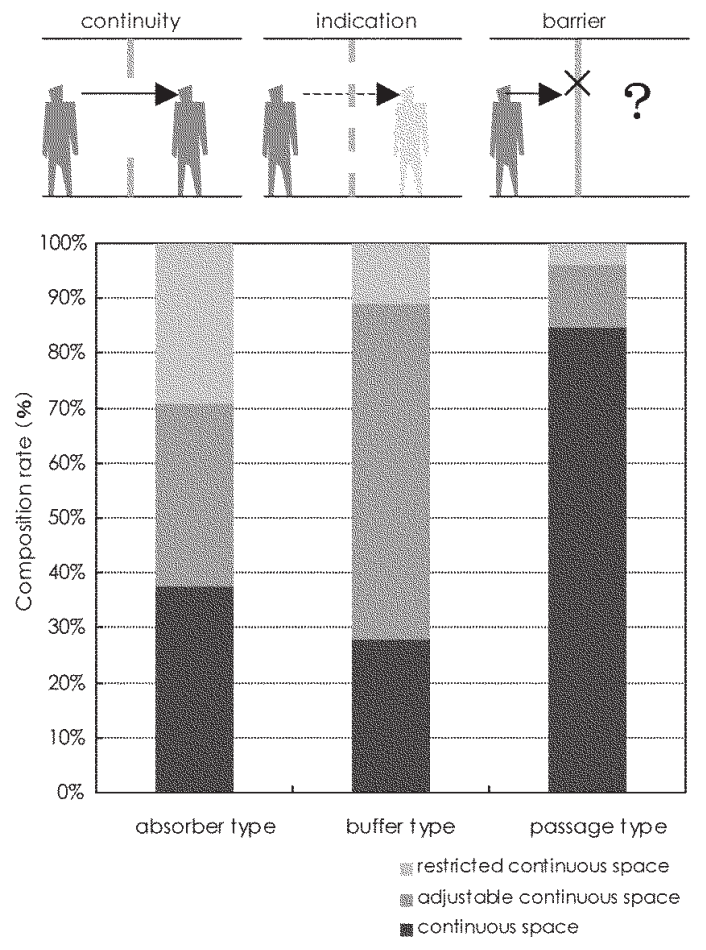

Fig.8. Integration by Sight

Table 2. Planning Factors for Zone Level Boundary Area

\begin{tabular}{|l|l|l|}
\hline \multicolumn{1}{|c|}{ Absorber type } & \multicolumn{1}{c|}{ Buffer type } & \multicolumn{1}{c|}{ Passage type } \\
\hline Clear articulation & Layout surrounded by the other zone & Parallel layout \\
\hline Locate at the deep circulation level & Multiplicity of adjacent zones & Obscure articulation \\
\hline & Flexible office furniture plans & Facing to the main traffic line \\
\hline & Multiple articulation techniques & A clear traffic line \\
\hline & A sense of being surrounded with open views & A good visibility \\
\hline & Combined traffic line of circulation level & \\
\hline & Adjustable continuous visibility & \\
\hline
\end{tabular}




\section{A Summary of the Case Study}

The case study was carried out in an office where one organization of a company had been occupied. The number of workers who own their personal desks and chairs were 19. The area of this office space is $254\left[\mathrm{~m}^{2}\right]$. It is shown in Fig.9. that there were four-zones planned in this office. 1) A personal-work zone where a person works at a personal space on his/her own desk and chair. 2) A creation zone where there is an enhanced meeting room with ICT tools. 3) An amenity zone for relaxation and refreshment, facilitated with different types of chairs and a table of a different height. And 4) An interaction zone where there is no function, but a place to activate workers communication. There is a table 9 meters long and two different types of chairs surrounding communication tools such as projectors, white-boards, etc.

We examined the office planning concepts and architectural factors for this office. With a comparison of boundary area types from our previous study, the characteristics we examined for this interaction zone were familiar with the planning factors of the Buffer type boundary areas. (Table 3.)

In this case study, we focused on the communication that occurred in this interaction zone in order to find out the worker's activities that took place in the buffer type boundary area. In addition, we verified the significance of the activities against their work processes through questionnaire surveys.

\section{Research and Analysis Methods}

The research at the office was carried out for five days (from the $29^{\text {th }}$ of Aug. 2004 to the $3^{\text {rd }}$ of Sept. 2004). All the communications and actions of workers happening in the office were recorded in notes with

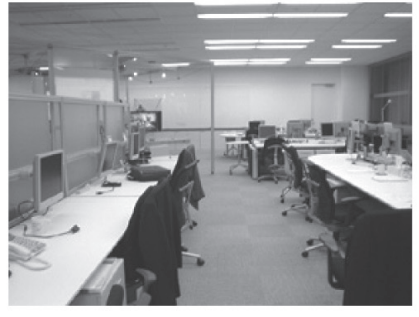

Personal-work zone

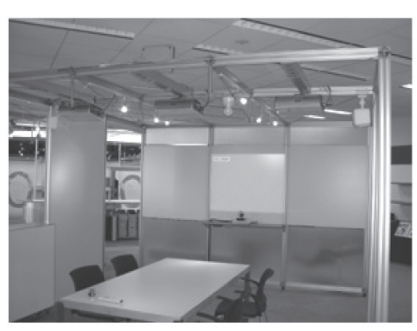

Creation zone

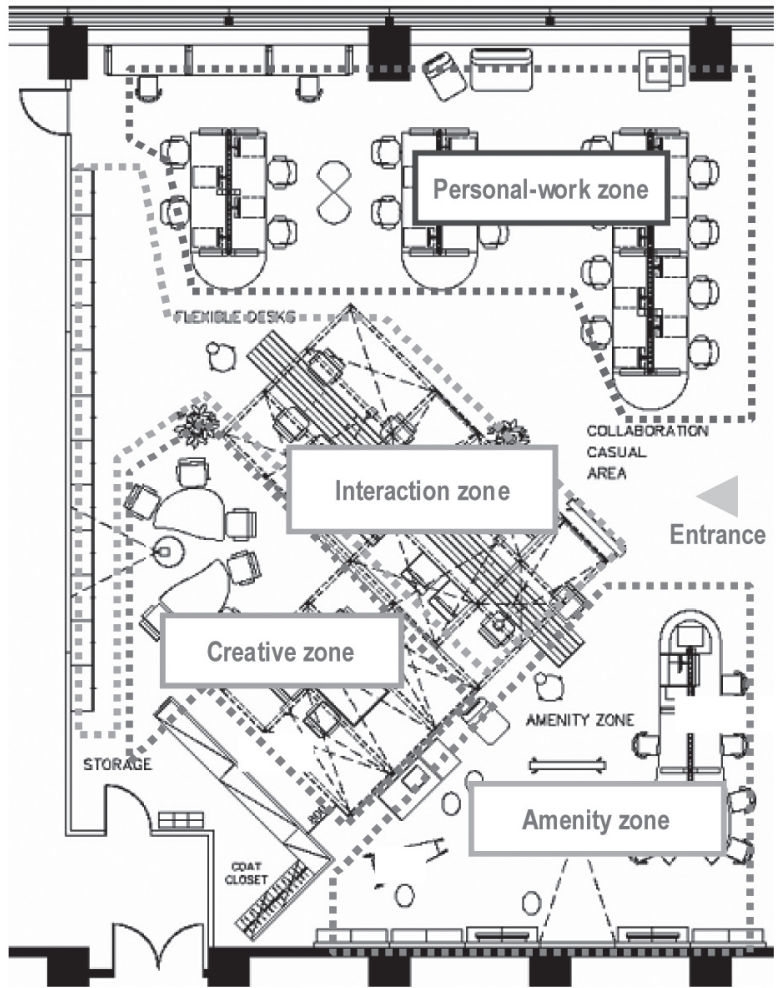

Fig.9. Plan for Case Study Office

photographs by two observers. To make the contents of the communications clear, the observer interviewed workers whenever activities took place. Additionally, all conditions of the office including solo-work were also recorded on video camera.

We created the data sheets and defined "Communication IDs" and "Communication scenes" as units to count the number of times communication occurred in the office. The definition is listed below.

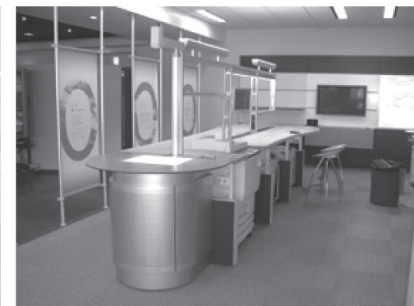

Amenity zone

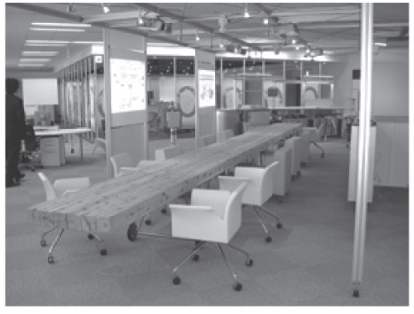

Interaction zone

Table 3. Planning Factors of the Buffer Type Boundary Area

\begin{tabular}{|l|l|}
\hline \multicolumn{1}{|c|}{ Characteristics of the interaction zone } & \multicolumn{1}{c|}{ Planning factors } \\
\hline $\begin{array}{l}\text { Placed in the center of the office space and surrounded by other } \\
\text { zones (Personal-work zone, creation zone, amenity zone) }\end{array}$ & Layout surrounded by the other zone \\
\hline $\begin{array}{l}\text { Adjoined with various zones (Personal-work zone and Creation } \\
\text { zone) }\end{array}$ & Multiplicity of adjacent zones \\
\hline $\begin{array}{l}\text { Utilizing office furniture that obscures space }(9[\mathrm{~m}] \text { long table that } \\
\text { is articulated with Pole) }\end{array}$ & Flexible office furniture plans \\
\hline $\begin{array}{l}\text { Various articulation techniques (design of wall, windows, pillars, } \\
\text { etc.) }\end{array}$ & Multiple articulation techniques \\
\hline Articulation technique with ceiling frame & A sense of being surrounded with open views \\
\hline Integrate primary traffic line and other traffic lines & Combined traffic lines of circulation level \\
\hline Adjustable visibility (roll screens and materials of walls) & Adjustable continuous visibility \\
\hline
\end{tabular}




\section{- Communication IDs:}

The number of conversations was divided by topics of the conversation. For example, both short conversations at the personal-work zone and long meetings at the creation zone are counted as one communication ID, because both have one topic for each.

- Communication scene:

The number of conversations was separated when either a tool, place or a person was changed. For example, if the conversation starts at a personal desk and then moves to the interaction zone to communicate particularity about the same topic, this conversation is counted up to two communication scenes due to the change of place and one communication ID because of the same topic. Additionally, if they used a white-board with this topic at the same zone, the communication should be counted as three communication scenes due to the tool used and one communication ID because of the same topic.

\subsection{Analysis and Findings}

Five features were observed from the perspective of communication as the activities in this interaction zone from this survey as shown below.

a) Group-work without reservations.

b) A person joins in the middle of a group-work.

c) Communications occur incidentally.

d) Solo-work and group-work in one area.

e) Relations between communication running times and the number of workers.

Based on these features, we verified the relation between the planning factors for the buffer type boundary areas and the activities that occurred in the area.

\section{a) Group-work without Reservations}

The interaction zone and the creation zone are used for group-work such as meetings or conferences. We counted the number of communication IDs as the number of meetings that was not reserved in the space. The incidence of such cases was $67 \%$ for the interaction zone and $43 \%$ for the creation zone.

In addition, when we asked in the interview about meeting space availability in this office, the worker answered that there are meeting places for about four people anywhere in this office without room reservations especially in the interaction zone.

From these results, the interaction zone can be the application for ad hoc communications and group-work. This indicates that group-work without reservation is activated by the planning factors of the buffer-type boundary area such as "a sense of being surrounded", "multiple articulation techniques" and "flexible office furniture plans".

\section{b) A Person joins in the Middle of a Group-work}

From the survey, the scene of a person joining into the middle of a group-work has been observed as a general characteristic of the interaction zone. For example, when group-work is going on and a person wants to have another person's opinion, they can ask someone else in the office casually, or someone who looks at the group-work scene and is aware of something good can join in and relate his information or idea to the group unexpectedly.

We counted the number of these incidents in each zone and compared them to see the relation between this activity and the architectural factors. As a result, the number of incidents at the interaction zone was the highest between zones, and the communication running time was long. In addition, these scenes at the interaction zone are mapped on the plane. (Fig.10.) This shows that a person joining into the middle of a group-work appeared between the interaction zone and other zones. This is because the interaction zone has open windows with easy access to other zones, in other words the "adjustable continuous visibility" is one of the planning factors.

\section{c) Communications occur Incidentally}

This can be seen when a worker goes to the bathroom, takes a coffee break or picks up stationery. The worker may be aware of something on his way and communicates with someone casually. We focused on these communications that took place at the interaction zone. The communications that occurred by chance were counted along with the number of the communication scenes. The highest occurrence of these incidents took place at the amenity zone, and the second was at the personal-work zone. Although the interaction zone did not show a high number of incidents, the position where the communication occurred in the interaction zone was characterized by the architectural factors of its zone.

Fig.11. shows the number of communication scenes that occurred at each position (MT1-A, MT1-B, MT2-A, MT2-B, MT3-A, MT3-B) with the bar chart. The communication happened at the side along the main corridor in the office (MT1-A, MT2-A and MT3-A). Additionally, the number of communications on the side close to the amenity zone is higher. It is likely that these results can be seen on the effect on the layout of the interaction zone that is next to the different level of the traffic lines.

\section{d) Solo-work and Group-work in One Area}

Fig.12. shows the fluctuation of the utilization for "solo-work", "communication" on group-works and "empty space" in each zone. These are confirmed with the video recorded every minute in the office.

In the personal-work zone, the solo-work took place continuously from 9:00 to 20:00 all day and many communications occurred for a short time. In the creation zone, the group-work mainly took place for a long time. The short time solo-work happened just before group-work started for the preparation of the meeting or happened just after group-work finished. The interaction zone showed medium circumstances 


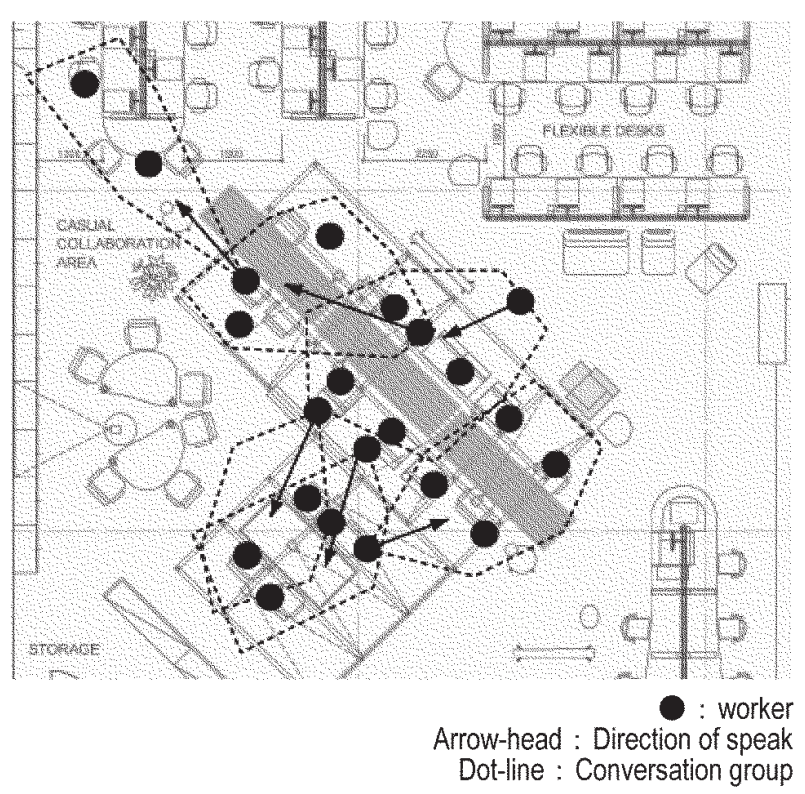

Fig.10. A Person Joins in the Middle of a Group-work

between these two zones. Group-work and solo-work often took place at the same time and at the same table that was facilitated by the 9 meters long table. Additionally, it was observed that the workers doing solo-work at the interaction zone seated on the chairs at the side having an open view to the office, and the workers doing group-work seated at the other side were turning their back to the office. All of this amounts to saying that the activity of the solo-work and groupwork in one area occurs because of "adjustable continuous visibility" and "flexible office furniture plans" of the planning factors.

e) Relations between Communication Running Times and the Number of Workers

The number of communication IDs observed was plotted and fitted on the line by the least squares method in each zone to compare the communication running times and the numbers of workers. (Fig.13.)

The lines for the personal-work zone and the amenity zone were positioned at a short period of the communication running times and small number of workers. The creation zone was positioned widely for the number of workers, but was consistent with the communication running times. The positioning for

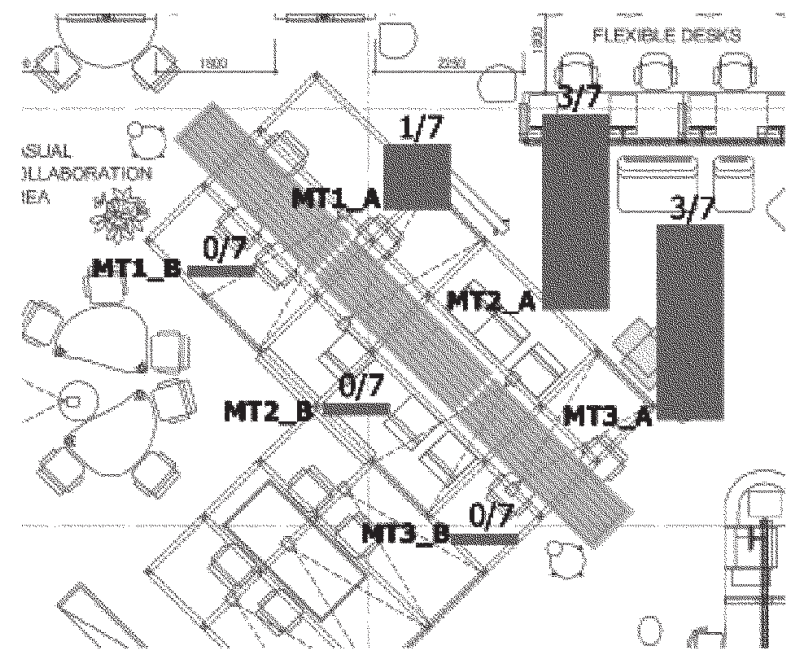

Numerator: The number of incidental communications on each point Denominator: The total number of incidental communications

Fig.11. Communications Occurring Incidentally

the interaction zone was in between them. Therefore, this interaction zone can diversify the communication between workers in the office. It is likely that these phenomena relate to the architectural factors of the layout surrounded by the other zone and the multiplicity of adjacent zones.

\section{Discussion}

In this case study, we extracted five features a) to e) as activities that take place in the office. It can be stated that all these activities occurred as a result of architectural composition of the boundary areas.

From this case study and former studies of office planning factors, we made Table 4 . for the relation between planning factors and activities that occurred in buffer type boundary areas. In addition, the questionnaire survey had been carried out to ask the importance of activities (five features: a) to e) in the case study) observed. More than $70 \%$ of workers indicated a positive answer. Therefore, it is not too far from the truth to say that the activities that occurred in buffer type boundary areas are important for the work processes.

This paper presents two issues to support the next stage of office planning in Japan.

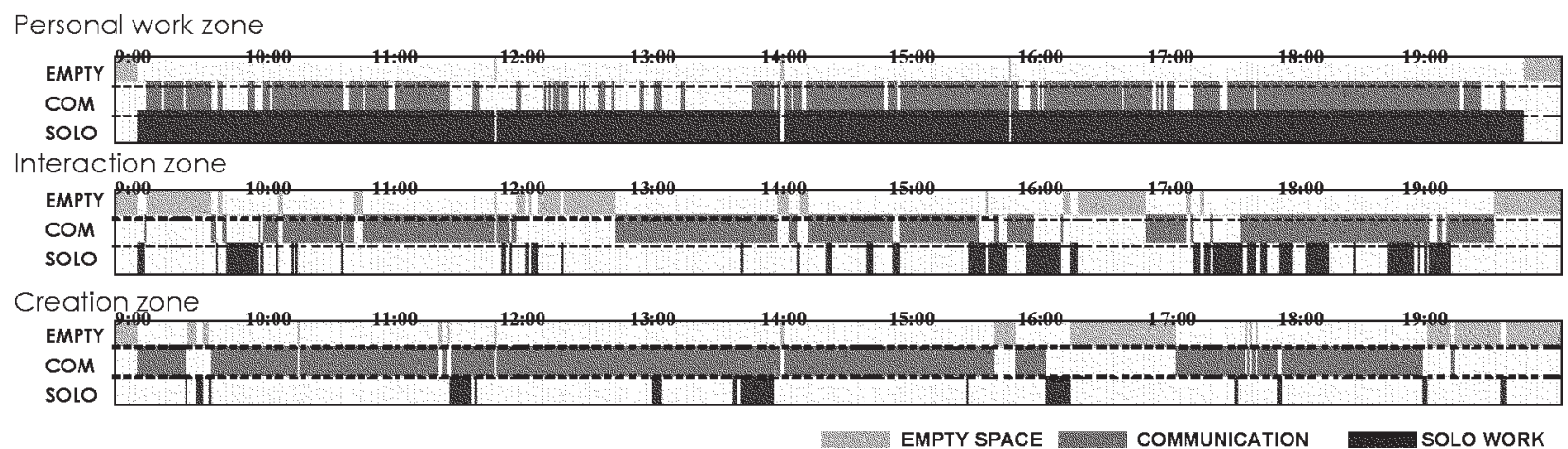

Fig.12. Fluctuation of Each Zone 


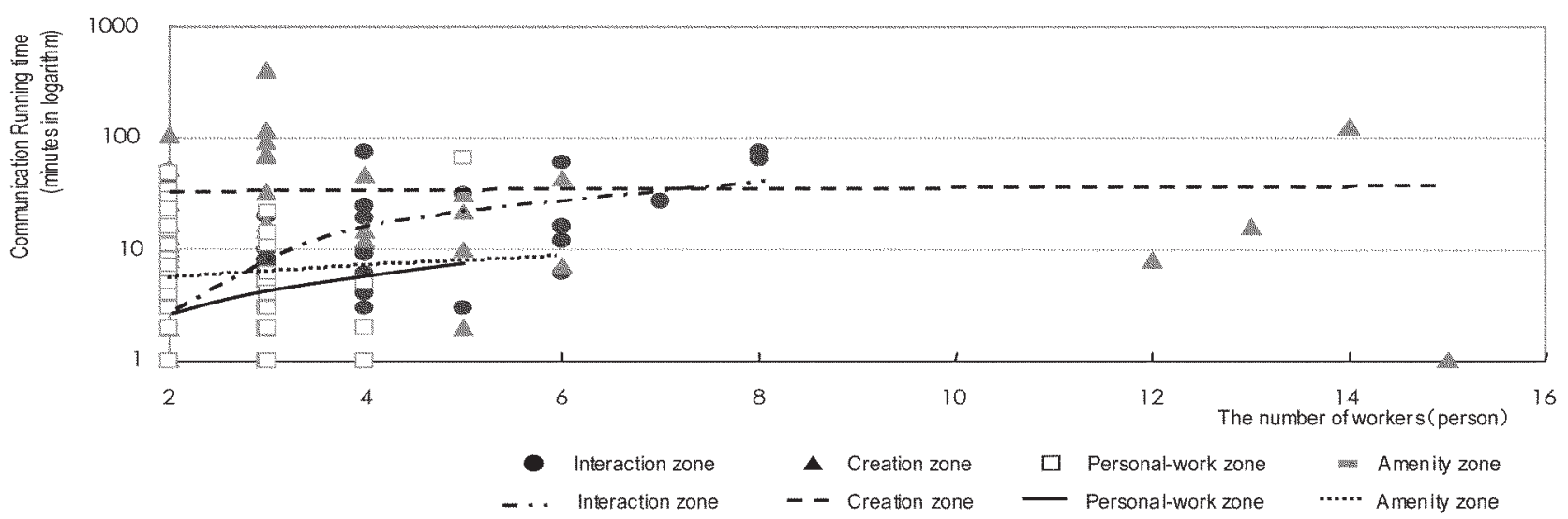

Fig.13. Communications in Each Zone

Table 4. Relation between Planning Factors and Activities which Occurred in Buffer Type Boundary Areas

\begin{tabular}{|l|l|}
\hline \multicolumn{1}{|c|}{ Planning factors } & \multicolumn{1}{c|}{ Activities } \\
\hline Layout surrounded by the other zone & Activate medium communications such as a short time meeting, ad-hoc meetings, etc. \\
\hline Multiplicity of adjacent zones & The communications between workers in office can be diversified. \\
\cline { 1 - 2 } Flexible office furniture plans & The communications take place incidentally. Solo-work and group-work occur in one area. \\
\cline { 1 - 2 } Multiple articulation techniques & \multirow{2}{*}{ Absorb the communications occurred incidentally. } \\
\cline { 1 - 2 } A sense of being surrounded with open views & Activate communications in office. The communications occur incidentally \\
\cline { 1 - 2 } Combined traffic lines of circulation level & $\begin{array}{l}\text { A person joins in the middle of a group-work. Solo-work and group-work take place in one } \\
\text { area. }\end{array}$ \\
\cline { 1 - 2 } Adjustable continuous visibility &
\end{tabular}

1. The classification of the zone level boundary areas, which is one of the trends for office planning in Japan. (Table 2.)

2 . The relation between planning factors of buffer type boundary areas and activities that take place in this area. (Table 4.)

Future studies might consider examining activities occurring in "absorber type" and "passage type" boundary areas. Furthermore, the planning factor for zone level boundary areas can be developed considering organization and work-style.

\section{References}

1) Naka, R. and Yamaguchi, S. (2002) A Study on the Tendency of Indicator Value of Office Area for Space Management, Journal of Architecture, Planning and Environmental Engineering No.551, p. 135 .

2) Shimoda, S., Iki K., Morozumi, M. and Shiota, M. (1997) Analysis Method on Space Evaluation Considering Office Worker's Attributes and Sections' Physical Environments. Study on facility management system for Japanese industry, Journal of Architecture, Planning and Environmental Engineering No.499, p.245.

3) Shimoda, S., Iki, K., Morozumi, M. and Shiota, M. (2000) Development of Analysis Method for the Improvement of Office Standard by Worker's Evaluations. Study on facility management system for Japanese industry NO.3, Journal of Architecture, Planning and Environmental Engineering No.533, p.275.

4) Shimamura, H., Yamada, T., Sugiyama, T. and Iwata, Y. (1998) A Study on the Free-Address Process of Research Office Part I. The effect of a new type free address office analyzed by user satisfaction evaluation, Journal of Architecture, Planning and Environmental Engineering No.509, p.129.

5) Mori, A., Tsunekawa, K., Kato, A. and le Roux, P.C. (2002) A Study on Relationships among Plan Compositions, Work-Styles, and Communication Behaviors in Office, Journal of Architecture, Planning and Environmental Engineering No.551, pp.129.
6) Aoki, Y. and Yuasa, Y. (1993) Private Use and Territory in AlleySpace Hypotheses and tests of planning concepts through the field surveys on alley-space Part1, Journal of Architecture, Planning and Environmental Engineering No.449, pp.47-55. 